\title{
Gli autori di questo numero
}

\section{Guido Piero Alpa}

Professore ordinario di Diritto civile nell'Università "La Sapienza“ di Roma

\section{Claudia Burlando}

Ricercatore confermato presso il Dipartimento di Economia e metodi quantitativi nell'Università di Genova

\section{Albina Candian}

Professore ordinario di Diritto privato comparato nell'Università di Milano

\section{Giuseppe Carriero}

Docente di Diritto dei mercato finanziari nell'Università "Tor Vergata" di Roma

\section{Pierluigi Ciocca}

Economista e accademico dei Lincei

\section{Marco Confalonieri}

Professore associato di Economia aziendale nell'Università Cattolica di Milano

\section{Paolo Di Betta}

Professore associato di Economia e gestione delle imprese nell'Università di Palermo

\section{Carlo Giampaolino}

Professore ordinario di Diritto commerciale nell'Università "Tor Vergata" di Roma

\section{Gaia Greco}

Assegnista di ricerca presso l'IRAT CNR di Napoli

\section{Raffaele Lener}

Professore ordinario di Diritto privato comparato nell'Università "Tor Vergata" di Roma

\section{Pietro Masi}

Professore ordinario di Diritto commerciale nell'Università "Tor Vergata" di Roma 


\section{Gian Piero Milano}

Professore ordinario di Diritto canonico e diritto ecclesiastico nell'Università "Tor Vergata" di Roma. Preside della Facoltà di Giurisprudenza

\section{Giuseppe Niccolini}

Professore ordinario di Diritto commerciale nell'Università "Luiss Guido Carli" di Roma

\section{Lidia Noto}

Dottoranda di ricerca di Modelli per la programmazione e il controllo a supporto delle politiche e strategie nella Pubblica amministrazione nell'Università di Palermo

\section{Eugenio Picozza}

Professore ordinario di Diritto amministrativo nell'Università "Tor Vergata" di Roma

\section{Roberto Ruozi}

Professore emerito di Economia degli intermediari finanziari dell'Università "Bocconi" di Milano

\section{Ruggero Sainaghi}

Professore associato di economia aziendale nell'Università IULM di Milano

\section{Giuseppe Santoni}

Professore ordinario di Diritto commerciale nell'Università "Tor Vergata" di Roma

\section{Barbara Sibilio Parri}

Professore ordinario di Economia aziendale nell’Università di Firenze

\section{Andrea Todeschino}

Dottore in General management nell'Università di Genova 


\section{ECONOMIA E DIRITTO DEL TERZIARIO \\ Economia e Diritto del Terziario pubblica articoli che riguardano l'economia e il diritto nel comparto dei servizi}

\section{Processo di referaggio degli articoli proposti per la pubblicazione}

Gli articoli che sono proposti alla rivista per la pubblicazione sono sottoposti in forma anonima a due successivi livelli di referaggio.

Il primo livello di referaggio (di ammissibilità) viene effettuato sull'abstract dell'articolo dai Membri del Comitato di Direzione che ne valutano la congruità ai temi trattati della rivista.

Il secondo livello di referaggio (di pubblicabilità) viene effettuato sull'articolo da due referee scelti all'interno del Comitato di Direzione o all'esterno tra accademici, ricercatori, esperti di materie di economia, diritto e terziario, che ne valutano contenuto e forma.

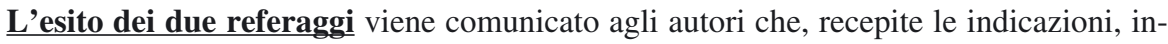
vieranno alla Segreteria di redazione il contributo per la pubblicazione.

Abstract e articoli devono essere inviati al dott. Matteo Paltrinieri dell'Ufficio Studi della Banca Carige all'indirizzo mail rivista.edt@carige.it Tel 010/5792266

Sarà compito della Segreteria di Redazione della rivista garantire l'anonimato dei contributi in sede di referaggio e mantenere i rapporti con gli autori.

\section{Norme per autori e collaboratori}

\section{L'ABSTRACT deve avere le seguenti caratteristiche:}

Per il primo referaggio (di congruità del tema trattato rispetto alla linea editoriale della rivista) si richiede un abstract di massimo 30 righe (300 parole) che in modo essenziale ma esaustivo presenti il lavoro secondo la seguente struttura:

- OBIETTIVI

- ASPETTI METODOLOGICI E DI QUADRO (metodo di analisi usato, ambiti settoriali e territoriali, arco temporale analizzato, limiti della ricerca, fonti utilizzate)

- CONTENUTO, da esporre preferibilmente seguendo la successione dei paragrafi

- RISULTATI E CONCLUSIONI

L'ARTICOLO deve avere le seguenti caratteristiche:

- lunghezza massima 20 cartelle (circa 2300 caratteri spazi esclusi a cartella).

- Il titolo dell'articolo deve essere sintetico e gli autori sono indicati con Nome e Cognome (sul nome degli autori viene richiamata in nota l'eventuale attribuzione dei paragrafi).

- I capitoli e i paragrafi sono numerati (introduzione e conclusioni escluse) con cifre arabe puntate.

- L'articolo deve essere preceduto da due brevi abstract (10-15 righe) uno in italiano e uno in inglese.

Norme redazionali generali

NUMERAZIONE DELLE PAGINE. Numerare progressivamente e visibilmente le pagine con cifre arabe. 
CAPOVERSI. Rientrare ad ogni capoverso servendosi del tasto tabulatore e non della barra spaziatrice.

MAIUSCOLE. Occorre attenersi alla massima uniformità per quanto riguarda l'uso delle maiuscole e delle minuscole, cercando di ridurre le prime all'essenziale.

Evitare le maiuscole per i termini comuni, aventi caratteristiche di generalità. Es: stato, governo, parlamento, regione, sindacato, vescovo, polizia, esercito, ecc.

Non usare la maiuscola per aggettivi e sostantivi che indicano nazionalità. Es: gli italiani, i formaggi francesi, ecc.

Nell'uso della terminologia politico amministrativa adottare il seguente criterio: ministero delle Partecipazioni statali, ministro della Pubblica istruzione, commissione Bilancio, ecc.

Nel caso di una locuzione in più termini usare la maiuscola soltanto per il primo. Es: Banca nazionale del lavoro, Parlamento europeo, Partito radicale, ecc.

Evitare assolutamente in ogni sede (testo, note, indice dei nomi) di scrivere il nome degli autori tutto in maiuscolo.

SIGLE. In tondo alto/basso e senza puntini tra una lettera e l'altra. Es. Bnl, Fiat, Pil, Usa, Acli, ecc.

CORSIVI. L'uso del corsivo è riservata ai termini stranieri, salvo quelli entrati ormai nell'uso comune (come élite, leader, partner). Le parole appartenenti al primo gruppo avranno il plurale della lingua cui appartengono, quelle appartenenti al secondo gruppo sono invece invariabili (le élite, i leader, i partner).

Fermo restando l'indicazione dei limitarne l'uso, si userà il corsivo per le parole che l'autore vuole evidenziare in particolare e per i volumi e gli articoli citati nel testo.

NERETTO. Non se ne fa assolutamente uso.

ACCENTAZIONE. Si raccomanda la correttezza nell'accentazione delle vocali: à,ì,ò,ù (sempre con accento grave); cioè, è (con accento grave); né, sé, perché, affinché, poiché, ecc (con accento acuto).

TRATTINI. I trattini che - come in questo caso - individuano un inciso sono preceduti e seguiti da spazio.

CONVENZIONI VARIE. Si raccomanda anche il rispetto di alcune convenzioni, come le seguenti:

- p. e pp. (e non pag. e pagg.); s. e ss. (e non seg. e segg.); cap.; cit.; cfr.; ecc.; vol. e voll.; n. e nn.;

- p. 24 e non p.24 (cioè con lo spazio); A. Manzoni e non A.Manzoni (cioè con lo spazio); J.M. Keynes e non J. M. Keynes (cioè senza spazio tra le due iniziali del nome);

- Cit. (in tondo); ibidem, passim, infra, supra (in corsivo).

\section{Note}

La numerazione delle note deve essere progressiva. Non sono ammesse note bis, ter, ecc..

OPERE CITATE

1. Se si tratta di un volume:

G. Pasquino, Modernizzazione e sviluppo politico, Bologna, il Mulino, 1970, II edizione, p. 9 (pp. 9-13, pp. 5 s., pp. 7 ss)

2. Se si tratta di un'opera tradotta:

M. Weber, Wirtschaft und Gesellschaft, Tubingen, Mohr, 1920; trad. it Economia e società, Milano, Comunità, 1968, vol. I, parte II, cap. V, p. 432.

3. Se si tratta di un contributo in un volume miscellaneo:

G. Pasquino, Lo sviluppo politico, in Antologia di scienza politica, a cura di G. Sartori, Bologna, il Mulino, 1970, pp. 419-472. 
4. Se si tratta di un articolo di una rivista:

M.M. Kellner, Democracy, in «Journal of politics», 37, 1975, n. 4, pp. 35-64.

5. Se si tratta di un'opera con un curatore:

Un sistema politico alla prova, a cura di M. Caciagli e A. Spreafico, Bologna, il Mulino, 1975.

6. Se si tratta di un'opera con più autori:

R. Rossi e B. Verdi, Storia economica ....

R. Rossi, B. Verdi e C. Neri, Storia economica ....

R. Rossi et al., Storia economica ....

\section{Riferimenti bibliografici}

Si usa la bibliografia cosiddetta tradizionale.

Seguire gli esempi di seguito riportati:

Colombo, R.M., Settecento senza amore: studi sulla narrativa inglese, Roma, Bulzoni, 1983.

Di Michele, L., L'educazione del sentimento, Napoli, Istituto Universitario Orientale, 1977.

Fiedler, L.A., Love and Death in the American Novel, New York, Dell, 1966; trad. it. Amore e morte nel romanzo americano, Milano, Longanesi, 1983.

Foster, J.R., The Abbé Prévost and the English Novel, in «PMLA», 42, 1927, pp 443464.

Garber, F., Meaning and Mode in Gothic Fiction, in Studies in Eighteenth Century Culture, a cura di H.E. Pagliaro, vol. III, Racism in Eighteenth Century, ClevelandLondon, Western Reserve University Press, 1973.

Lenoir, R. e Lesourne, J (a cura di), Où va l'Etat? La souveraineté économique et politique en question, Paris, Ed. Le Monde, 1992.

Manciulli, M., Podestà, L. e Ruggeri, F., Il dilemma organizzativo, Milano, FrancoAngeli, 1986.

Marradi, A., Boudon: un sociologo che ha sbagliato specializzazione, in « Rassegna Italiana di Sociologia», XXVIII, 1982, n. 3, pp. 345-398.

In appendice alla rivista si elencano gli Autori di questo numero con una sintetica qualifica professionale della principale attività svolta. Esempi:

Giorgio Bianchi

Professore ordinario di Diritto privato nell'Università "La Sapienza" di Roma

Giovanna Verdi

Professoressa di Economia e gestione delle imprese nell'Università Politecnica delle Marche

Mario Rossi

Ricercatore in Economia e gestione delle imprese nell'Università di Genova

Lucia Neri

Assegnista di ricerca presso l'Irat-Cnr di Napoli

Andrea Rosso

Nucleo per la ricerca economica della Banca d'Italia

Margherita Verde

Avvocato cassazionista della Banca d'Italia

Sonia Bianco

Dottore commercialista 